\title{
Preisträgerarbeit des SGP-Förderpreises 2020
}

\section{Marco Fresa, Oberarzt an der Universitätsklinik für Angiologie, CHUV \\ (Centre Hospitalier Universitaire Vaudois), Lausanne: Endovenous laser combined with Sclerotherapy for the treatment of large intramuscular venous malformations}

\section{Hintergrund}

Intramuskuläre venöse Malformationen sind eine relativ häufige vaskuläre Pathologie, für welche es bis heute keine standardisierte Behandlung gibt. Die Sklerotherapie funktioniert relativ gut, aber führt bei ausgedehnten Läsionen häufig zu unvollständigen Resultaten bzw. Rückfällen. Die chirurgische Exzision ist oft recht invasiv und kann bei ausgedehnten Fällen nur eine Teilentfernung der vaskulären Malformation anbieten.

In den wissenschaftlichen Guidelines wird daher auch die endovenöse Thermoablation intramuskulärer venöser Malformationen empfohlen, ohne dass dazu jedoch wissenschaftliche Standards existieren.

Ziel dieser Arbeit soll es sein, die Wirksamkeit einer kombinierten Therapie mit endovenöser Thermoablation und Sklerotherapie bei intramuskulären venösen Malformationen prospektiv zu studieren und zu dokumentieren.

\section{Material und Methoden}

Dieses Projekt wurde 2018 ausgearbeitet und vom Ethikkomitee des Kantons Waadt bewilligt. Seit Januar 2019 werden Patienten eingeschleust. Die ersten 7 Fälle wur- den bereits als kleine Fallserie zur Publikation eingereicht.

Für die vorliegende Arbeit werden alle behandelten Patienten von Januar 2019 bis Juni 2021 prospektiv eingeschleust, vollständig dokumentiert und bezüglich des Behandlungserfolgs analysiert.

Aus den ersten 7 zur Publikation eingereichten Fällen lässt sich vorläufig zusammenfassen, dass durch die kombinierte Anwendung der endovenösen Thermoablation mit Schaumsklerotherapie in $100 \%$ ein vollständiger Verschluss der ausgedehnten intramuskulären venösen Malformationen erreicht werden konnte. Bei den Kontrolluntersuchungen nach einer Woche und nach 6 Wochen fanden sich im Ultraschall keine komprimierbaren venösen Kammern. Nach 6 Monaten waren bei allen Patienten die Malformationen um 60-100\% des ursprünglichen Volumens reduziert.

Bis Mitte 2021 rechnen die Autoren damit, über 30 Patientinnen und Patienten in die Studie eingeschlossen und analysiert zu haben. Das primäre Ziel des Projekts besteht in der Analyse der mittelfristigen Erfolgsrate (Verschlussrate und Volumenreduktion nach 6 Monaten). Als sekundärer Endpunkt wird die Verbesserung der Lebensqualität gemessen. Die detaillierte Darstellung der
Subtypen von ausgedehnten intramuskulären venösen Malformationen soll ferner aufzeigen, für welchen Typen welche Technik die besten Resultate erzielt.

\section{Einsatz der zugeteilten Mittel der Schweizerischen Gesellschaft für Phlebologie}

Die Fördergelder von 10000 CHF werden für die Entschädigung des Ethikkomitees (5000 CHF), für die Entschädigung des Datenmanagements und die Entschädigung der Statistiker eingesetzt.

\section{Präsentation der \\ Studienresultate:}

Die Studienresultate sollen voraussichtlich anlässlich des 21. USGG-Jahreskongresses vom 02.-03.12.2021 in Interlaken präsentiert werden.

\section{IMPRESSUM}

Verantwortlich für Mitteilungen der SGP:

Prof. Dr. med. Jürg Hafner, Zürich 